\title{
Restoration of Legacy Parallelism: Transforming Pthreads into Farm and Pipeline Patterns
}

\author{
Vladimir Janjic $^{1} \cdot$ Christopher Brown $^{2}$ (D) $\cdot$ Adam D. Barwell $^{3}$
}

Received: 20 November 2020 / Accepted: 4 May 2021 / Published online: 10 June 2021

(C) The Author(s) 2021

\begin{abstract}
Parallel patterns are a high-level programming paradigm that enables non-experts in parallelism to develop structured parallel programs that are maintainable, adaptive, and portable whilst achieving good performance on a variety of parallel systems. However, there still exists a large base of legacy-parallel code developed using ad-hoc methods and incorporating low-level parallel/concurrency libraries such as pthreads without any parallel patterns in the fundamental design. This code would benefit from being restructured and rewritten into pattern-based code. However, the process of rewriting the code is laborious and error-prone, due to typical concurrency and pthreading code being closely intertwined throughout the business logic of the program. In this paper, we present a new software restoration methodology, to transform legacy-parallel programs implemented using pthreads into structured farm and pipeline patterned equivalents. We demonstrate our restoration technique on a number of benchmarks, allowing the introduction of patterned farm and pipeline parallelism in the resulting code; we record improvements in cyclomatic complexity and speedups on a number of representative benchmarks.
\end{abstract}

Keywords Parallel patterns · Restoration - Pthreads - Program transformation · Refactoring · Code analysis · Farm · Pipeline $\cdot$ TBB

Christopher Brown

cmb21@st-andrews.ac.uk

Vladimir Janjic

vjanjic001@dundee.ac.uk

Adam D. Barwell

a.barwell@imperial.ac.uk

1 School of Science and Engineering, University of Dundee, Dundee, UK

2 School of Computer Science, University of St Andrews, St Andrews, UK

3 Department of Computing, Imperial College London, London, UK 


\section{Introduction}

Parallel patterns are a well-established high-level parallel programming model for producing portable, maintainable, adaptive, and efficient parallel code. They have been endorsed by some of the biggest IT companies, such as Intel and Microsoft, who have developed their own parallel pattern libraries; e.g. Intel TBB [35] and Microsoft PPL. A standard way to use these libraries is to start with a sequential code base, identifying in it the portions of code that are amenable to parallelisation, together with the exact parallel pattern to be applied. Proceeding with instantiating the identified pattern at the identified location in the code, after possibly restructuring the code to accommodate the parallelism. Sequential code therefore gives the cleanest starting point for introduction of parallel patterns. There exists, however, a large base of legacy code that was parallelised using lower-level, mostly ad-hoc parallelisation methods and libraries, such as pthreads [12]. This code is usually very hard to read and understand, is tailored to a specific parallelisation, and optimised for a specific architecture, effectively preventing alternative (and possibly better) parallelisations and limiting portability and adaptivity of the code. An even bigger problem, from a software engineering perspective, is the maintainability of the legacy-parallel code: commonly, the programmer who wrote it is the only one who can understand and maintain the code. This is due to both complexity of lowlevel threading libraries and the need for custom-built data structures, synchronisation mechanisms, and sometimes even thread/task scheduling implemented in the code. The benefits of using parallel patterns lie in a clear separation between sequential and parallel parts of the code and a high-level description of the underlying parallelism, making the patterned applications much easier to maintain, change, and adapt to new architectures. In this paper, we deal with farms and pipelines. In a farm, a single computational worker is applied to a set of independent inputs. The parallelism arises from applying the worker to different input elements in parallel. In a parallel pipeline, a sequence of functions, $f_{1}, f_{2}, \ldots, f_{m}$ are applied to a stream of independent inputs, $x_{1}, \ldots, x_{n}$ where the output of $f_{i}$ becomes the input to $f_{i+1}$; the parallelism arises from executing $f_{i+1}\left(f_{i}\left(\ldots f_{1}\left(x_{k}\right) \ldots\right)\right)$ in parallel with $f_{i}\left(f_{i-1}\left(\ldots f_{1}\left(x_{k+1}\right) \ldots\right)\right)$. In this paper, we present a new methodology for the restoration of legacy-parallel code into an equivalent patterned form, through the application of a number of identified program transformations; the ultimate goal of which is to provide a semi-automatic way of converting legacy-parallel code into an equivalent patterned code, therefore increasing its maintainability, adaptivity, and portability whilst either improving or maintaining performance. The transformations presented in this paper are intended as manual transformations. We envisage incorporating implementations of these refactorings into a semi-automated refactoring tool as future work.

This paper makes the following specific research contributions:

1. we present a novel software restoration methodology for converting legacyparallel applications into their structured (patterned) parallel equivalents, via the farm and pipeline patterns; 
2. we present a new set of (manual) restoration transformations that attempt to systematically, (i) eliminate pthread operations from legacy $\mathrm{C} / \mathrm{C}++$ programs; (ii) perform code repair, fixing any bugs introduced in $i$; and, (iii) reshape code in preparation for parallel pattern introduction;

3. we evaluate these transformations on a set of benchmarks, demonstrating that removal of parallelism can allow us to manually derive structured parallel code that is comparable to the original legacy-parallel version in terms of performance, while being more portable, adaptive, and maintainable.

\section{Software Restoration}

In this section, we propose a new Software Restoration methodology for improving the structure of legacy-parallel $\mathrm{C}++$ code by applying a series of incremental program analysis and transformation steps to rewrite the code into its patterned equivalent. Software restoration is based on program transformation and code analysis and aims to:

1. discover the instances of common patterns in legacy-parallel code;

2. eliminate undesirable legacy parallel primitives from the same code; and

3. replace the removed parallel primitives with instances of parallel patterns.

The input to the Software Restoration process is a legacy-parallel $\mathrm{C} / \mathrm{C}++$ program that is based on some low-level parallelism library, such as pthreads, and the output is a semantically-equivalent $\mathrm{C} / \mathrm{C}++$ program based on parallel patterns. In this way, we obtain well-structured code based on a higher level of parallel abstraction, which is significantly more maintainable and adaptive while still preserving good performance of the original, highly-tuned parallel version. In this paper, we will

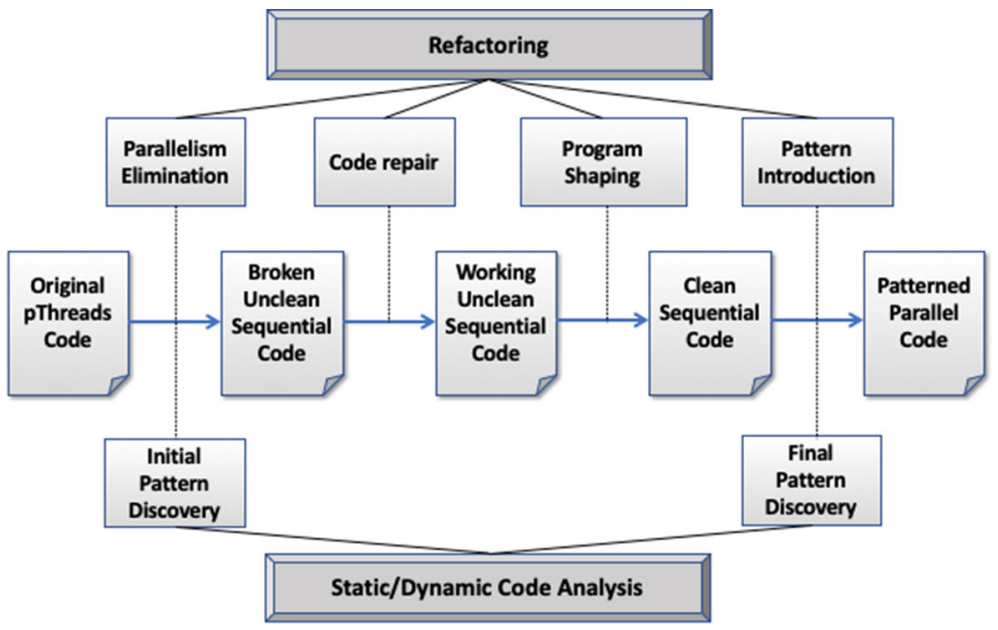

Fig. 1 Software restoration process 
focus on the TBB library as our target code. It is important to note, however, that transforming the code into a patterned form also increases the portability of the code and gives a wider opportunity for parallelisation using different techniques, libraries and pattern approaches. In this paper, we target TBB as just one example of a typical and common pattern library but the patternisation step could easily be replaced with other equivalent and more general frameworks; e.g. the Generic Reusable Parallel Pattern Interface (GrPPI) [18], which allows multiple different pattern backends to be targetted from a single interface. Indeed, prior work on refactoring to introduce GrPPI [8] patterns could easily be deployed at this stage, further increasing portability of the patterned code.

The Software Restoration methodology consists of a number of steps, each applying a class of code transformations, some of which are driven by the pattern discovery code analysis. The whole process is depicted in Fig. 1. In the below description, we will focus on the code transformation steps. We will use a synthetic, but representative, parallel pipeline as a running example in order to demonstrate the transformation. Listing 1 presents aspects of the original parallel code with pthreads that are pertinent to this demonstration. 


\section{Listing 1: Original Simple Pipeline Code}

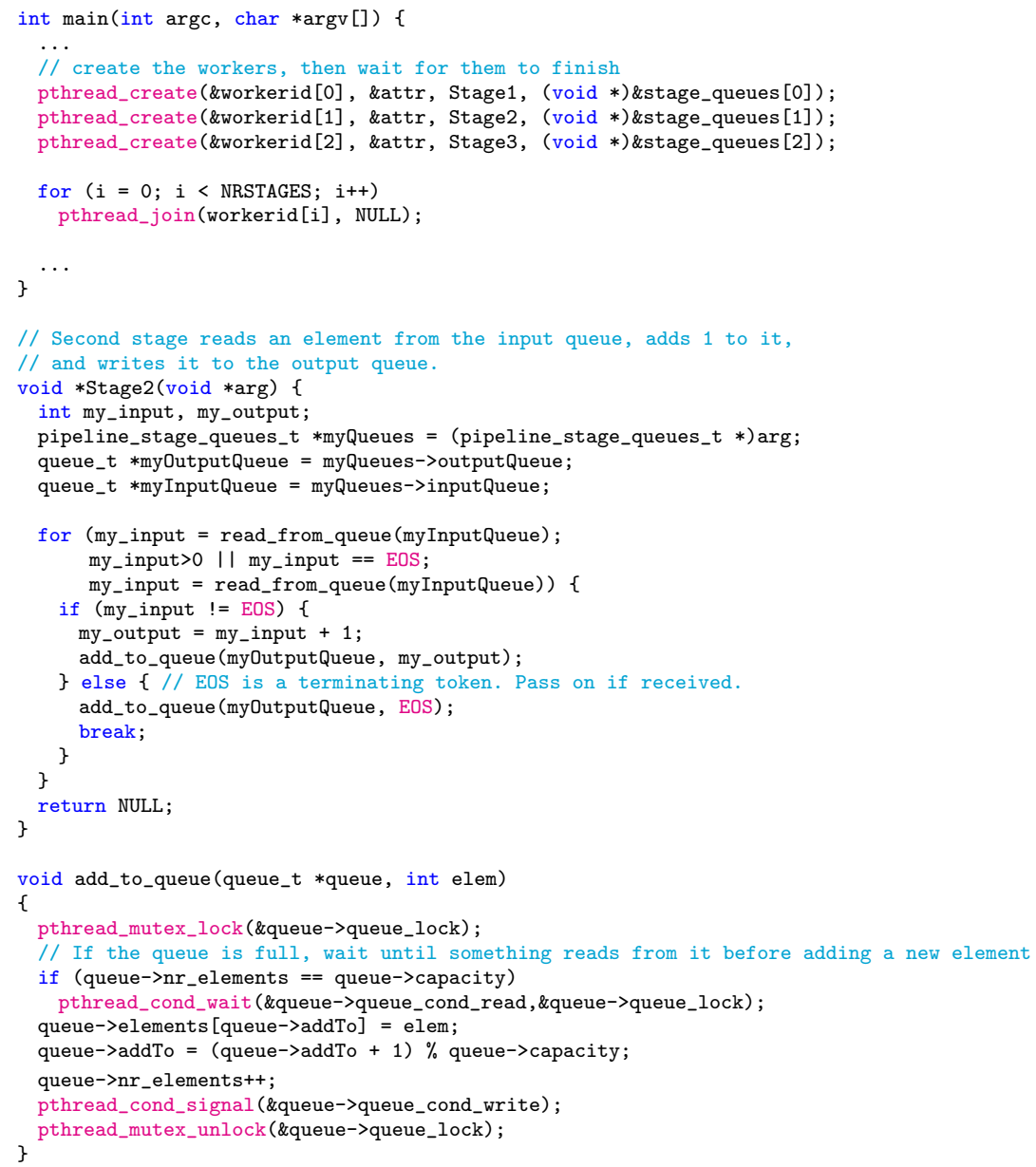

In the above main function (Lines 1-12), a pipeline of three stages is created using three threads. The stages are connected by queues such that all stages have an output queue, and stages two and three have an input queue. After creation, the main function waits for the threads to finish their work (Lines 8-9) before continuing. In Lines 14-34, we show the function for the middle stage of the pipeline, which reads an integer from the input queue, increments it by one, then puts it into the output queue. The first and third stages have a similar structure, where the first stage acts as a source of integers for the second stage, and the third stage doubles its inputs before adding them to the final output queue. All the relevant synchronisation code for the queues can be found in two functions: add_to_queue and read_from_queue. Only add_to_queue (Lines 36-47) is shown here; read_from_queue is defined similarly. Both functions use one mutex lock and two conditional variables. The latter are used for 
synchronisation when threads are waiting to insert an element into a full queue or for reading from an empty queue (e.g. at the start of the program). When a thread needs to add to the queue, it first acquires the queue lock and checks if the queue is full (Lines 38-41). When the queue is full, the thread releases the lock and waits for a signal that some other thread has consumed an element of this queue (queue$>$ queue_cond_read conditional variable, Line 41). After this conditional variable is signalled, the thread enqueues the element, updating the queue counter and pointer in the process (Lines 42-44). Finally, the thread signals that an element has been added to the queue (queue->queue_cond_write conditional variable, Line 45) and releases the queue lock (Line 46) before returning.

\subsection{Parallelism Elimination}

The initial step, Initial Pattern Discovery, analyses the original pthreaded code and discovers those parts of it, if any, that correspond to instances of parallel patterns. In our example, this stage identifies the linear pipeline created in Lines 4-6, with the pipeline stages being the functions: Stage1, Stage2, and Stage3. This process could be achieved by using a technique similar to the one described in [10] Following pattern discovery, the first code transformation step is applied, where pthread operations and primitives are either removed or transformed, eliminating parallelism. In Listing 1, this impacts main and add_to_queue. Listing 2 shows the resulting code.

Listing 2: Simple Pipeline Code with Parallelism Removed

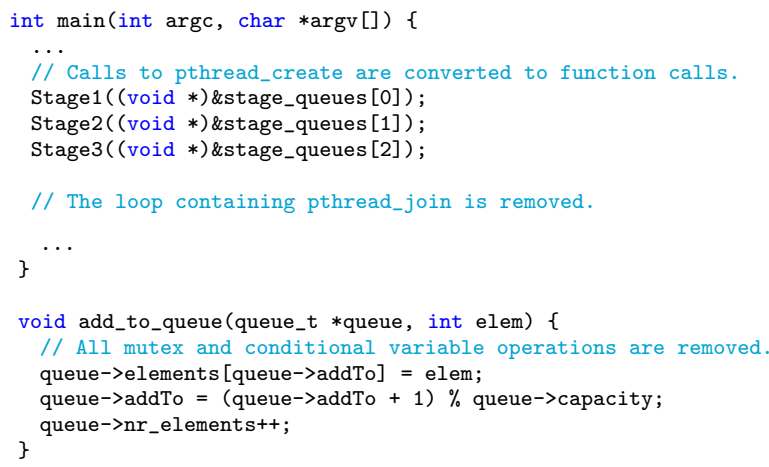

We note that the Parallelism Elimination stage does not guarantee that a program's functional behaviour is preserved and thus errors may be introduced. Here, Stage1 contains a for-loop that enqueues elements in its output queue. Since the second stage, which reads from that queue, is no longer consuming those elements concurrently, and the queue is smaller than the total number of elements produced, the second stage will now consume and process only a subset of its inputs in the original pthreaded version after Stage1 returns. Because the semantics of the program have changed following Parallelism Elimination, the code must be repaired. 


\subsection{Code Repair}

Our example is just one of many in which merely removing pthread constructs introduces errors (see Sect. 5 for more examples). The next step in Software Restoration is, therefore, to repair the potentially broken code produced by Parallelism Elimination. In general, due to the potential complexity of this repair stage, multiple transformations may need to be applied. In order to effect repairs in our running example it is necessary to stop the first stage from overflowing its output queue. This can be achieved by merging the loops found in Stage1, Stage2, and Stage3, thereby resulting in a loop where the operations in stages two and three are applied to each integer produced by stage one in the same iteration that produces it. Listing 3 represents the result of this process, where Stage1, Stage2, and Stage3 are first lifted into a new function, Pipe, and subsequently unfolded (i.e. unfolding in the transformational sense, à la Burstall and Darlington [11]). The for-loops exposed by this unfolding are then merged, allowing all three stages to be executed within a single iteration. This avoids the first stage overflowing its output queue, and consequently, results in a program that is sequential but semantically equivalent to the original pthreaded program.

Listing 3: Simple Pipeline Code after Code Repair

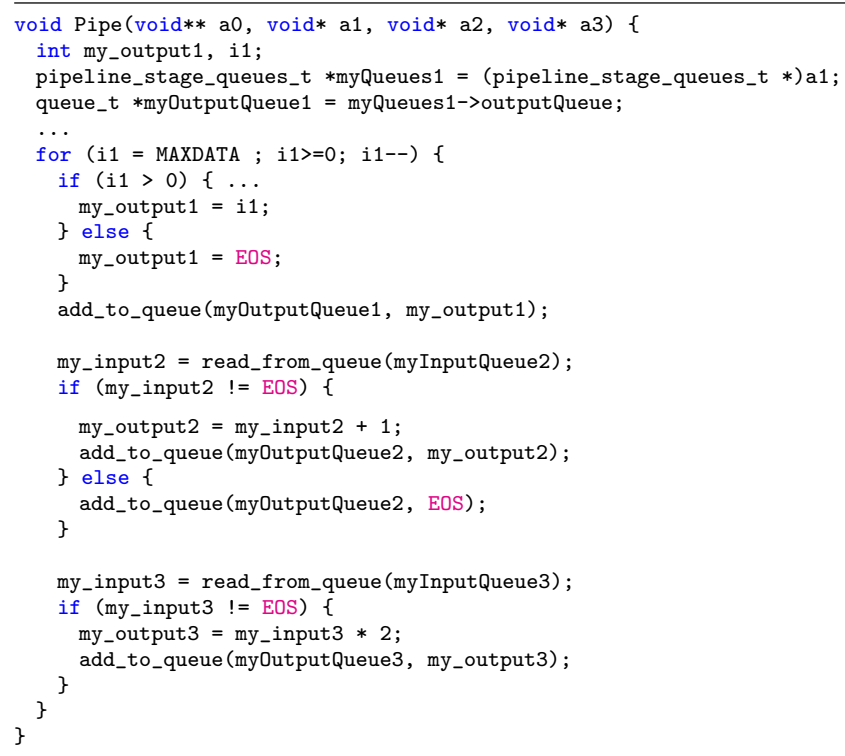

\subsection{Program Shaping}

The code produced by the Code Repair stage may still contain artefacts from the original legacy parallelisation. In our running example, this includes the EOS token and intermediate queues. In other examples, custom-built representations of flat data 
structures, e.g. arrays, introduced for chunking purposes may also be present. These artefacts are redundant and could hinder alternative (and possibly better) parallelisations of the code. The next step is, therefore, to eliminate residual artefacts of legacy parallelism, and to improve structure where such improvements make the code more amenable to the introduction of patterned parallelism. As in Code Repair, due to the potential complexity of this task, multiple transformations may need to be applied. Each Program Shaping refactoring results in a program that is semantically equivalent to the one it transforms. The result of the Program Shaping stage on our running example can be found in Listing 4, where both the EOS token and intermediate queues have been removed (see Sect. 4.3 for details) and the individual stages lifted back into functions.

Listing 4: Clean Sequential Simple Pipeline Code

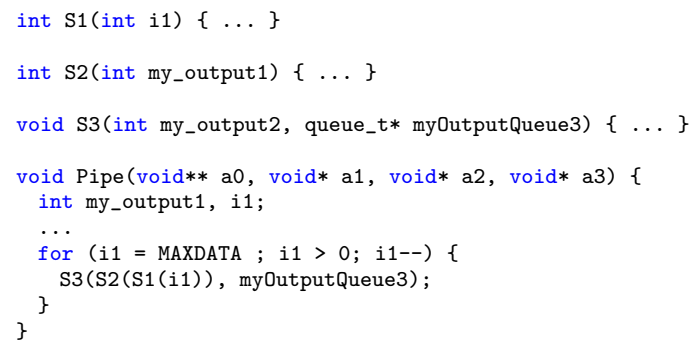

\subsection{Pattern Introduction}

After the final pattern discovery analysis is performed and the final patterns to be introduced are identified, together with the locations in the code where this will be done, the final step is to introduce instances of parallel patterns into the now-clean sequential code. The parts of the sequential code are replaced by calls to the functions from the high-level pattern libraries such as Intel $T B B$ [35] or OpenMP [16]. This results in final, patterned parallel code that is semantically equivalent to the starting legacy-parallel code, but with much cleaner structure and simpler, higher-level code that allows easier maintainability, adaptivity and portability.

\section{Pipeline Assumptions}

In this paper, we demonstrate our methodology on a subset of pipelines defined using pthreads. Whilst the refactorings described below apply only to this subset, they can be extended to facilitate a more general application of the restoration process. We assume that a valid pipeline (i.e. a pipeline that can be restored using the below refactorings) is linear, that all tasks are generated by the first stage, and that no subsequent stages will create or destroy tasks. Moreover, we assume that the first stage of the pipeline will produce an end-of-stream (EOS) token, which is 
propagated through the pipeline, and results in a stage halting when it is received as input. A valid pipeline is assumed to be set up in a single function containing a sequence (or loop) of pthread_create calls. For each pthread_create call there must be a corresponding pthread_join call in the same function. We assume that each stage of a pipeline is run on a single thread. However, no assumptions are made regarding threnad attributes, arguments passed to the function upon thread creation, or the second argument passed to pthread_join. Tasks are sent between stages in pipelines via intermediate queues. Each stage of a pipeline is assumed to have an input queue, $q_{1}$, and an output queue, $q_{2}$, given that $q_{1} \neq q_{2}$ and the output queue of stage $i$ is the input queue of stage $i+1$. Pipeline stages may only communicate via these intermediate queues; for simplicity, we assume that (intermediate) stages do not access global variables. Queues are assumed to be cyclic. If the queue is full, and the implementation does not wait for an element to be read before adding a new element, it is assumed that the queue overwrites (unread) elements. We assume that queues use pthread_cond_wait, pthread_cond_signal, pthread_mutex_lock, and pthread_mutex_unlock operations only. These restrictions are to ensure that, following Parallelism Elimination, the behaviour of the pipeline breaks in a consistent way. Any queue update functions should not be recursive. Whilst we make no assumption on the size of queues, the interesting case is when queues are smaller than the total number of tasks passing through the pipeline. Each stage of a valid pipeline is assumed to contain a loop that retrieves input and produces a modified version of it as output.

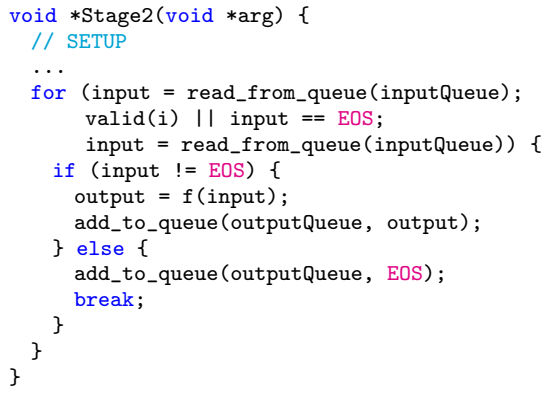

The for-loop is assumed to read input from the stage's input queue for each iteration, where the test expression is a disjunction permitting both EOS token and valid inputs (for some definition of valid). The test expression may be simplified by treating the EOS as a valid input. The body of the loop is assumed to comprise an ifstatement that checks for the EOS token, represented here by a preprocessor macro. When the input is not the EOS token, it is modified (Line 8) and added to the output queue (Line 9). Conversely, when the input is the EOS token, it is propagated to the next stage (Line 11) and a break statement used in order to halt the stage (Line 12). Should the EOS token be handled incorrectly and not halt the stage, due to our assumptions on the nature of the intermediate queues, the for-loop will wait indefinitely for input that will never arrive. Whilst we permit the occurrence of 
break statements only in the locations specified above, we assume that no part of the pipeline has continue or goto statements.

The first stage differs in that the input is not retrieved from an input queue, but tasks are generated in a for-loop. For example, in Stage1

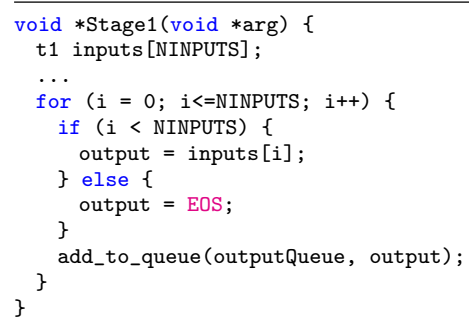

an array of inputs is iterated over and each element is sent to the second stage of the pipeline. We assume that the for-loop in the first stage is finite and that termination is controlled by the test expression and not through a conditional statement in the body of the loop. Once all tasks have been generated, the first stage will emit an EOS token and no further iterations of the loop occur.

\section{Restoration Transformations}

The following transformations are grouped according to the stages in Sect. 2 and all apply to $\mathrm{C}$ programs that adhere to the assumptions in Sect. 3. In this paper, we do not attempt to prove that our transformations preserve functional behaviour; indeed, some intentionally do not. Such proofs are left to future work, where they can be properly explored in depth. Instead, and where expected, we conjecture that our transformations preserve functional behaviour when they are applied to code that both adheres to the assumptions in Sect. 3 and that meets the pre-conditions of the individual refactoring. For example, commutativity of loops in Merge for-loops (Sect. 4.2). It is our intention that these assumptions and pre-conditions are sufficiently strong so as to render post-conditions and dynamic correctness checks unnecessary. Proof that this property holds for our refactorings is outside of the scope of this paper and will be considered as part of future work alongside proofs of general soundness.

In addition to the following transformations, standard refactorings may also facilitate the restoration process. For instance, the transformation to unfold a function definition [11] is used in both Code Repair and Shaping stages; e.g. in the former, it allows loops to be merged, and in the latter, it allows the elimination of intermediate queues. The extract method [21] transformation can be similarly used to lift a pipeline into a self-contained function, or to lift its individual stages (back) into separate functions. 


\subsection{Parallelism Elimination}

Parallelism Elimination comprises a single composite transformation that either removes or transforms pthread operations. As noted in Sect. 2, Parallelism Elimination does not guarantee that the result of the transformation will be semantically equivalent to the transformed program. It is applied to the functions that are identified as part of the valid pipeline and effects the following transformations.

- Removes \#include <pthread> when all pthread operations within the file are found within the functions identified as part of the valid pipeline.

- Removes all pthread operations within the pipeline functions aside from calls to both pthread_join and pthread_create.

- Removes all variable declarations whose types are defined as part of the pthread library, excepting pthread_t. This includes global declarations when those variables occur solely within the identified pipeline functions.

- Declarations in the form pthread_t $t$; are transformed into void* $t$;. As above, in the case where such declarations are global, the variables may occur only in the pipeline functions.

- Calls to pthread_create of the form,

1 pthread_create $(t, a, f, x)$

are transformed into the form:

$1 \quad t=f(x) ;$

Recall that Parallelism Elimination converts the type of pthread_t variables to void* variables of the same name(s), and that pthread_create requires that $f$ returns a value of type void*.

- Calls to pthread_join are transformed according to whether the second argument is NULL. When the second argument is not NULL, e.g. pthread_join $(t, x)$, the join operation is transformed into the form $x=t$. Otherwise, the call to pthread_join is removed.

- In cases where a call to pthread_join or pthread_create forms the righthand-side of an assignment statement, e.g.

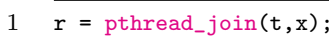

in addition to the transformation of the pthread operation, an assignment statement is inserted where the variable being assigned, $r$, is assigned the value of a successful call to the original pthread operation, here pthread_join and 0 . The assignments resulting from the transformation is: 
- Any for-loop whose body contains no statements following the removal of a pthread operation will itself be removed.

- Any if-statement with a branch whose body contains no statements following the removal of a pthread operation will be transformed to have only the other branch, or itself removed, if no such branch exists. For instance, given the for-loop from the synthetic pipeline example in Listing 1 ,

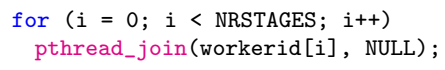

since the second argument to pthread_join is NULL, the join operation result is itself a statement, and the body of the for-loop contains no other operations, this for-loop is removed.

\subsection{Code Repair}

In addition to unfolding and extract method refactorings, the merging of loops is a key transformation of the Code Repair stage when restoring valid pipelines. Parallelism Elimination can result in one or more intermediate queues to overwrite elements before they can be read. Merging the queues of all pipeline stages ensures that no queue overflows. Whilst we only describe the merging of for-loops, a similar approach can be used to merge equivalent loop kinds.

Merge for-loops. A sequence of $n$ for-loops, in the same compound statement can be merged such that the result is a single loop containing the bodies of the original loops in the same order that they appeared in the original sequence. Any statements that appear in between loops in the original code, must be commutative with respect to all preceding loops; i.e. it must be possible to swap the ordering of the statements and preceding loops without changing the behaviour of the program. 
Listing 5: Intermediate Code Repair Stage for Simple Pipeline Example

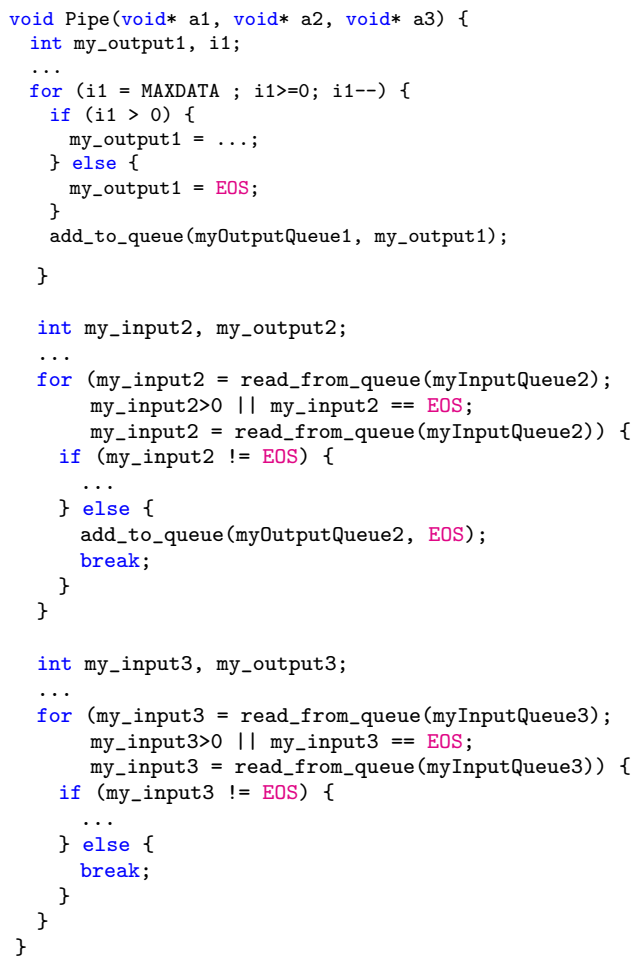

Listing 5 builds on the example in Listing 2, where the calls to Stage1, Stage2, and Stage3 have been lifted into the function Pipe using extract method and then unfolded. It is possible to merge all three loops since the statements in between the loops can be safely executed prior to the first and second loops. 
Listing 6: Following Merging of loops in Listing 5

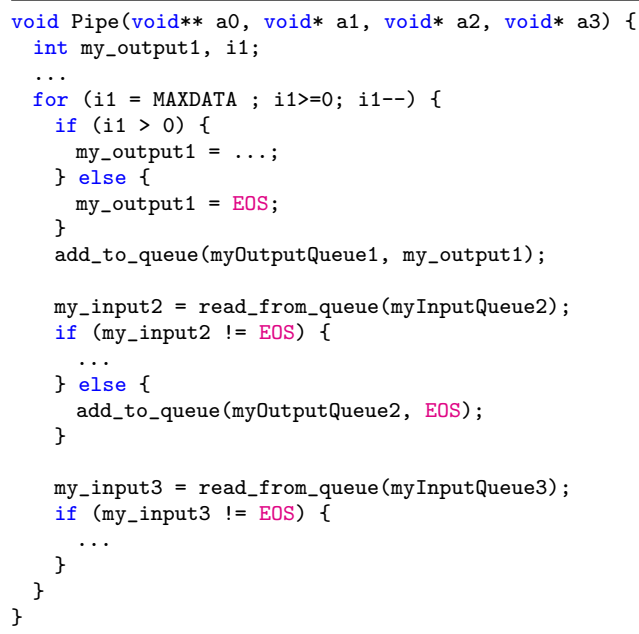

Since we assume that new tasks are only produced by the first stage and that all subsequent stages neither generate nor destroy tasks, it follows that the number of iterations for the first loop will be equal to the number of iterations for subsequent loops in the original pipeline. Consequently, the initialisation statement, test expression, and iteration expression of the merged loop will be those of the first loop; here, i1 = MAXDATA, i1 $>=0$, and $i 1--$, respectively. The bodies of the individual loops are included in the same order as the original loops themselves. Since the merged loop uses its initialisation statement, test expression, and iteration expression, the body of the first loop is included unchanged. Bodies of subsequent loops, however, are preceded by their update statement. For example, the body of the second loop is preceded by the assignment to my_input2 on Line 12 in Listing 6 which is taken from the update statement on Line 18 in Listing 5. This ensures that the input queue for each stage is read from only when a task has been added to that queue by the preceding stage. In addition to the inclusion of the update expression, the break statements from the original for-loops are removed, leaving the propagation of the EOS token in all but the final stage. In the final stage of our pipeline (Lines 19-22) we remove the entire else branch in Listing 6 since it contains only the break statement on Line 34 in Listing 5. Whilst the removal of these break statements is not strictly necessary, since they will only be evaluated when the first stage emits an EOS token once all other tasks have been processed, they are removed because they are redundant now that the termination of the merged loop is controlled by the first stage of the pipeline, which will terminate after generating the EOS token.

\subsection{Program Shaping}

Program Shaping represents the broadest stage in the process and presents the programmer with the largest range of choices in terms of transformations that may 
be effected. In addition to unfolding definitions and creating new functions via extract method, other standard transformations may be applied, e.g. dead-code elimination [28], in order to improve or simplify the structure of the code. In order to remove aspects of the code that represent optimisations enacted for the legacy parallelisation, both existing and novel transformations may be necessary. Novel transformations may include the unchunking of data, the removal intermediate, and now redundant, queues between stages, and a removal of the EOS token. In line with our running example, we propose transformations to remove EOS tokens and to remove intermediate queues.

\subsection{Remove EOS Token from Merged Loops}

Intuitively, we assume that Remove EOS Token from Merged Loops applies to the result of Merge Loops. Since termination of all stages is controlled solely by the merged loop, the EOS token is redundant and can be removed so that the resulting restored pipeline doesn't perform unnecessary work. By our assumption, at the beginning of the for-loop, there is an if-statement that determines whether the iterator being generated by the first stage is genuine output or the EOS token. This if-statement is replaced by the branch of the if-statement that produces genuine output. Additionally, the test expression of the merged loop is replaced by the condition of the if-statement being removed. This results in the merged loop executing one fewer iteration and the first stage of the (now-removed) pipeline no longer adding the EOS token to its output queue. For all other stages of the pipeline, we replace each stage's if-statement with their output branches, thus removing the EOS token behaviour.

Listing 7: Following application of Remove EOS Token from Merged Loops to Listing 6

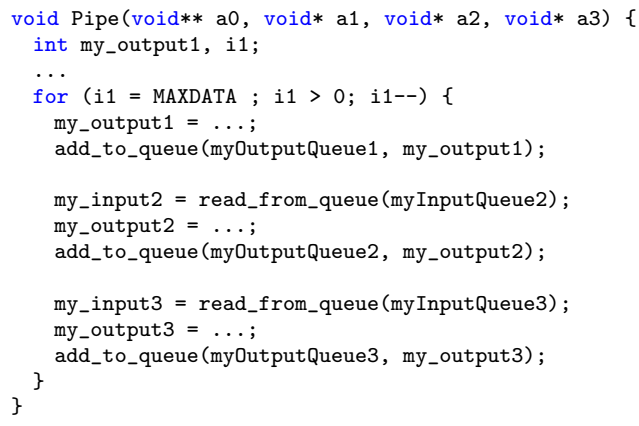

Listing 7 gives the result of applying Remove EOS Token from Merged Loops to the code in Listing 6. Here, the original test expression of the merged loop, $i 1>=0$ (Line 4 Listing 7), has been replaced with the condition of the if-statement from the first stage of the pipeline, $i 1>0$ (Line 5, Listing 7). That if-statement has itself been replaced by its true branch. The if-statements for the other two stages (Lines 
13-17 and 20-22, Listing 7) have similarly been replaced by their true branches, since their false branches handle the EOS token.

\subsection{Remove Intermediate Queues}

Following the application of Merge Loops the intermediate queues become redundant. They can be removed by inspecting, matching, and transforming read, write, and update operations pertaining to those queues. In our recurring example we begin this process having removed the EOS token, and having unfolded both add_to_queue and read_from_queue operations. Note that the add_to_queue operation in the third stage is not unfolded since this is the output of the pipeline itself.

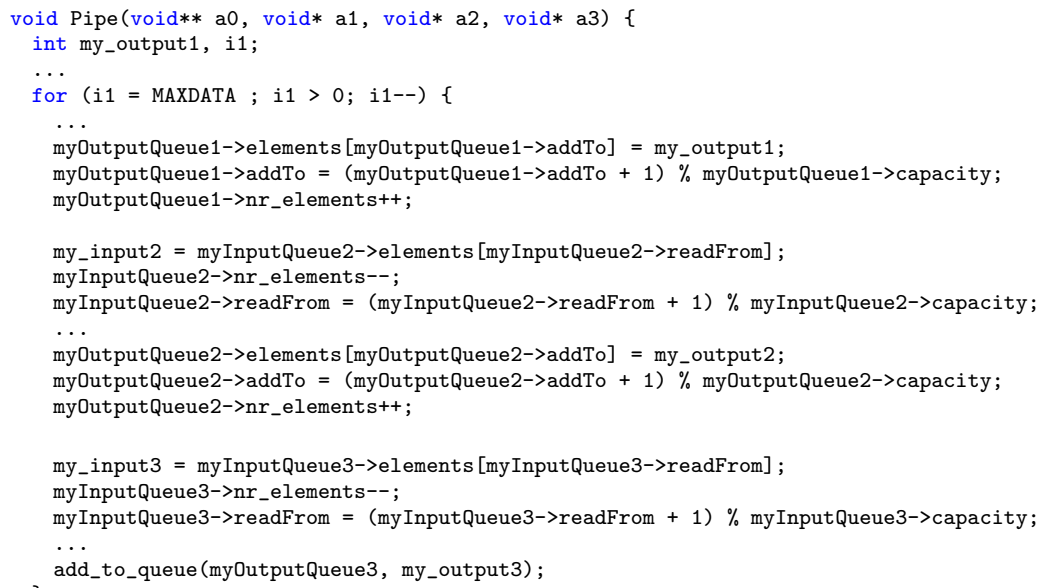

A variable is read from when that variable occurs in a statement and that variable is not being updated; e.g. capacity on Line 7 above. Similarly, a variable undergoes a write when it is being assigned to and is not being updated; e.g. elements in the first output queue is written to on Line 6. Finally, a variable is updated when it occurs in a statement that is both reading from and writing to that variable; e.g. addTo in Line 7 above. Basic increment operators, e.g. nr_elements ++ on Line 8 , are similarly considered updates due to their semantics. In order to transform these read, write, and update operations, we pair the operations in the order that they appear in the code and according to the variables they read, write, or update, and transform those pairs according to their composition. If two queues are semantically the same but referred to by different variables then they themselves will be considered the same during pairing; e.g. myOutputQueue1 and myInputQueue2 refer to the same intermediate queue, thus myOutputQueue1->elements and myInputQueue2->elements are similarly considered to be the same variable for pairing. In the above example, two cases arise: 
1. Updates to variables that do not occur elsewhere in the code pertain to queue housekeeping operations are therefore removed. In the above code, Lines 7, 8, $11,12,15,16,19$, and 20 are all removed.

2. A write followed by a read is merged into a single assignment statement s.t. the RHS of the read is replaced with the RHS of the write, and where the original write statement is removed. For example, in the above code, the write to elements on Line 6 and the read from elements on Line 10 can be paired (due in part to the behaviour of the queue reading the element that has just been added). Since this represents passing my_output 1 on Line 6 to my_input 2 on Line 10, it is possible to remove Line 10 and transform Line 6 into the form my_input_2 = my_output_1.

An unpaired read that is part of an update, e.g. capacity on Line 7, or a paired write, e.g. addTo on Line 6, is removed or otherwise transformed along with the update or paired write statement. Similarly, an unpaired read that is part of a paired read statement, e.g. readFrom on Line 10, is also transformed according to the paired read statement. When applied, the above transformations result in the removal of the two intermediate queues.

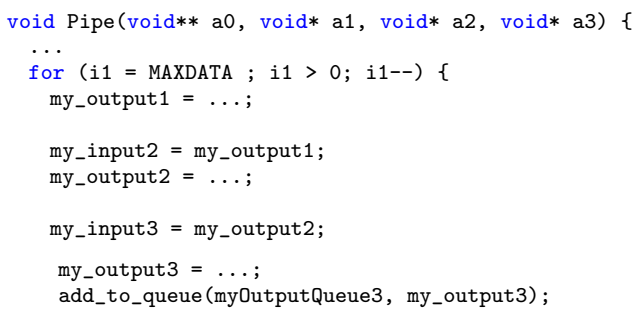

\section{Evaluation}

In this section, we present an evaluation of our restoration methodology on a number of examples of pthreaded $\mathrm{C}$ and $\mathrm{C}++$ applications taken from a variety of domains, including image convolution, nqueens, cholesky decomposition, blackscholes, pgpry, mandelbrot and matrix multiplication ${ }^{1}$. For each benchmark we evaluate the effectiveness of our technique using standard metrics, such as McCabe's Cyclomatic Complexity [31], lines of code and difference in runtimes between the original pthread version and the restored TBB version, using the maximum number of available cores; these results are summarised in Table 1, which also labels if each benchmark is a standard task from implementation $(\mathrm{F})$ or a pipeline $(\mathrm{P})$, where each stage can also be farmed. All of our execution experiments are executed 5 times and conducted on a server with Intel Xeon E5-2690 CPU with

\footnotetext{
$\overline{1}$ Repository of examples available at https://github.com/Paraformance/restoration
} 
Table 1 Metrics for each benchmark, where $\mathrm{F}=$ Farm, and $\mathrm{P}=$ Pipeline; performance times are in seconds on a 28 -core machine

\begin{tabular}{|c|c|c|c|c|c|c|c|}
\hline \multirow[t]{2}{*}{ Benchmark } & & \multicolumn{2}{|c|}{ McCabe } & \multicolumn{2}{|l|}{ Lines } & \multicolumn{2}{|c|}{ Performance (std dev) } \\
\hline & & Before & After & Before & After & Before & After \\
\hline Blackscholes & $\mathrm{F}$ & 29 & 29 & 366 & 393 & $38.5(0.07)$ & $39(0.42)$ \\
\hline MatMult & $\mathrm{F}$ & 9 & 15 & 176 & 146 & 918.7 (24.6) & $922.24(30.42)$ \\
\hline Mandelbrot & $\mathrm{F}$ & 12 & 11 & 145 & 142 & $2.21(0.01)$ & $2.27(0.04)$ \\
\hline Cholesky & $\mathrm{F}$ & 31 & 19 & 321 & 226 & $16.97(0.07)$ & $17.08(0.02)$ \\
\hline NQueens & $\mathrm{P}(2)$ & 41 & 24 & 421 & 337 & $8.63(0.04)$ & $8.622(0.27)$ \\
\hline PGPry & P (2) & 23 & 19 & 210 & 243 & $138.1(0.23)$ & $131(0.10)$ \\
\hline ImageConv & $\mathrm{P}(1)$ & 71 & 29 & 714 & 280 & $12.85(6.08)$ & $5.2(0.02)$ \\
\hline
\end{tabular}

The number of tokens for TBB pipelines are shown in parentheses

28 cores, running at $2.6 \mathrm{GHz}$ with $256 \mathrm{~GB}$ of RAM, with the Scientific Linux 6.2 operating system.

\subsection{Image Convolution}

Image Convolution is a technique widely used in image processing applications for blurring, smoothing, and edge detection. We consider an instance of the image convolution from video processing applications, where we are given a list of images that are processed by applying a filter. Applying a filter to an image consists of computing a scalar product of the filter weights with the input pixels within a window surrounding each of the output pixels:

$$
\operatorname{out}(i, j)=\sum_{m} \sum_{n} \operatorname{in}(i-n, j-m) \times \operatorname{filt}(n, m)
$$




\section{Listing 8: Original Convolution with PThreads}

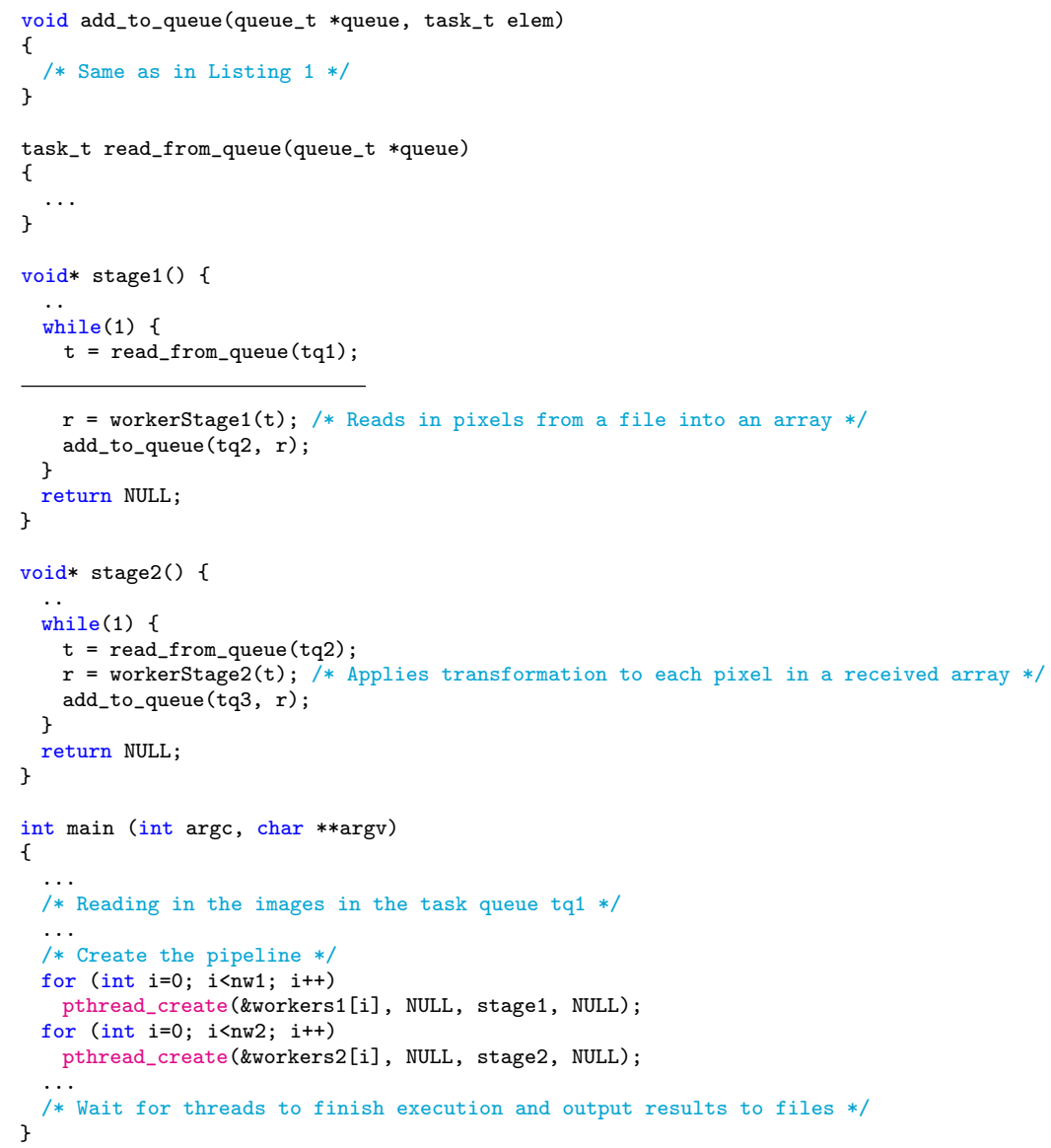

For the convolution example, we start off with a pthreaded version in Listing 8, with a similar structure as the other pipelined examples in this paper, and outlined in Sect. 2. After setting up the task queue for the first stage of the pipeline (e.g. by reading a list of names of files with images), the example creates the pipeline in Lines 37-40, spawning a number of worker threads for each stage of the pipeline. The pipeline stages are shown at Lines 11 and 21, respectively; each stage has a similar structure: a non-terminating while loop that retrieves a task from the stage's input queue (tq1 and tq2 for stage1 and stage2, respectively), computes the unit of work on the task item (Lines 15 and 25) and then places the result on an output queue (Lines 16 and 26). Functions add_to_queue and read_from_queue put a task in an output queue and read a task from an input queue, respectivelly, in a thread safe manner. The code for add_to_queue was shown in Listing 1. 
The first step to restoration is to remove the threading code; this is a fairly straightforward process, but results in an executable that no-longer terminates. This is due to the fact that there is no termination condition of the while loops within the stages. A simple repair for this step is to add a termination token, EOS. When no more tasks are available on the original input queue, EOS is sent through the pipeline, terminating the stages (Listing 9).

Listing 9: Convolution, Repaired with a Termination Token

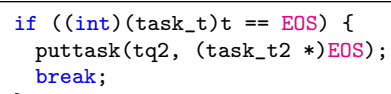

The next step is to perform program shaping which goes through various steps, including unfolding the various calls to gettask and puttask in the stages, merging the stages together, and finally removing the intermediate queue between the two stages (leaving the input and output queue; see Listing 10).

Listing 10: Stages merged, unfolded and intermediate queue removed

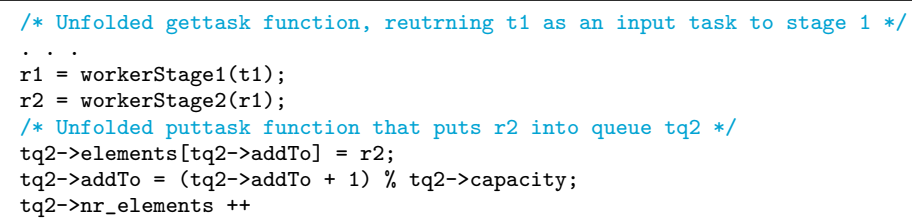

The final step in the shaping process is to arrive at the code shown in Listing 11 where we remove the input and output queues completely, and transform the program into a simple function composition; the function composition has been unfolded into the original for loop (Line 37-40 from Listing 8), and the loops merged into a single loop.

Listing 11: Convolution Shaped

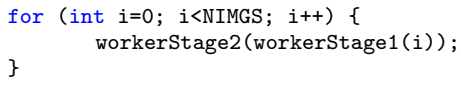

Finally, the fully shaped program from Listing 11 can be parallelised using a structured pattern approach. Here we use TBB, to define a pipeline, using $\mathrm{C}++$ classes, as shown in Listing 12.

Listing 12: Convolution Restored with TBB

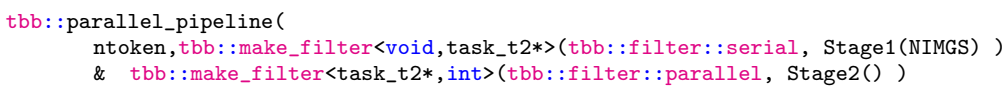




\subsection{Discussion}

Table 1 shows the summary of our results for all the benchmarks. For all benchmarks we see comparable results in the McCabe metrics, where the TBB version gives a better result, apart from Blackscholes, where the complexity is equal, and Matrix Multiplication, where the complexity actually increases. This is most likely because both of these benchmarks are simple farms, and the TBB logic actually introduces some complexity over simply calling pthread_create multiple times. The number of lines of code for the TBB version is mostly comparable, with most benchmarks showing a decrease in lines of code. Blackscholes shows a slight increase in LOC, most likely, again, due to the slight increase in code logic for TBB versus the pthread version. In terms of performance, again, the TBB versions are mostly comparable, with the exception of a few cases. For convolution, the TBB version performs $2.4 \times$ faster, due to the pthreading version introducing extra overheads in the locking code; Blackscholes also performs very slightly worse, by $1.28 \%$.

\subsection{Limitations}

The methodology presented in this paper is still preliminary and intended to be a foundational step to a more advanced (semi-automated) restoration technique that applies to many different kinds of patterns and applications. As such, we have noted a number of general limitations to the approach, which we document here.

As the transformations are currently applied manually, with the lack of a (semiautomated) tool, mistakes can easily happen as part of that manual transformation. The functionality of the transformed program has to be tested and checked with the original version at each step to ensure validity. However, if a (non-specialised) developer was undertaking this task, without any real direction or structure to parallelise their code, the manual process would undoubtedly take much longer and be a much more error-prone process. Our methodology aims to give a guide to programmers so that they can restore their programs, and act as a basis for future implementation efforts.

Furthermore, many code bases (including PARSEC) are large and diffuse, containing many files and many hundreds or thousands of lines of code. Applying such a manual technique to large and complex code is a very time consuming process, limiting our choice in use-cases and examples. Knowledge of the use-case is also needed in order to transform it correctly, taking into account subtle structural and algorithmic properties of the underlying source code. Often the most time consuming part of the process is understanding the underlying algorithmic properties, and not in the transformations themselves.

Our methodology only currently applies to farms and pipelines. A preliminary study of other applications with instances of different types of patterns, such as BSP, reduce, and map, seem to indicate that many of the restoration steps will be different, resulting in different transformation steps depending on the pattern instance to be restored. However, many of the tranformations that we present in this paper to eliminate pthreaded code can still be applied. We intend to pursue this direction as an avenue for future work. 


\section{Related Work}

The concept of a systematic, or structured approach to software restoration has, to our knowledge, been largely previously unexplored. A concept that is probably most related to software restoration is that of reverse engineering, which is a technique used to retrieve high-level requirements from existing sequential code [14, 15]. Yu et al. [37] proposed a technique that attempts to use refactoring to try and recover requirements goal models from legacy code. However, the work only targets sequential code and only capture high-level information that is not useful for parallel restoration. Refactoring has roots in Burstall and Darlington's fold/unfold system [11], and has been applied to a wide range of applications as an approach to program transformation [32], with refactoring tools a feature of popular IDEs including, i.a., Eclipse [36] and Visual Studio [33]. Previous work on parallelisation via refactoring has primarily focussed on the introduction and manipulation of parallel pattern libraries in $\mathrm{C}++[9,27]$ and Erlang [5, 7]. Another approach has been the automated introduction of annotations in the form of $\mathrm{C}++$ attributes [19]. Dig proposed an approach to parallelise loops in Java [20], but did not use highlevel algorithmic skeletons. Aldinucci and Danelutto proposed an approach to convert between skeleton configurations and could be used to introduce parallelism, but where the sequential program must also be defined using (sequential) skeletons [1]. Thompson et al. [29] proposed an approach to refactor sequential Erlang programs into concurrent versions, using program slicing to guide the refactoring process. However, their approach was not focussed on parallel performance, and did not use restoration or parallel patterns. High-level parallel patterns, sometimes known as algorithmic skeletons offer high-level abstraction over low-level concurrency methods [3, 22]. A range of pattern/skeleton implementations have been developed for a number of programming languages; these include: RPL [27]; Feldspar [4]; FastFlow [2]; Microsoft's Pattern Parallel Library [13]; and Intel's Threading Building Blocks (TBB) library [35]. Since patterns are well-defined, rewrites can be used to automatically explore the space of equivalent patterns, e.g. optimising for performance [24, 30] or generating optimised code as part of a domain-specific language (DSL) [23]. Moreover, since patterns are architecture-agnostic, patterns have been similarly implemented for multiple architectures [26, 34]. SPar [25] is a C++ internal domain-specificlanguage (DSL) for supporting the development of classic stream parallel applications targetting a FastFlow [2] backend. SPar allows the programmer to annotate $\mathrm{C}++$ code with high-level annotations, relating to the streaming and staging properties of the underlying algorithm. A compiler then transforms the SPar annotations into FastFlow code which can then be executed. We believe SPar would be a viable framework to support the introduction of patterns in our methodology. The P3ARSEC benchmark suite [17], offer patterned implementations of the Parsec benchmark suite using pattern-based parallel programming, in contrast to the standard Parsec implementations of pthreads, OpenMP and TBB. P3ARSEC instead uses a FastFlow backend, and makes use of several parallel patterns, including pipeline, farm, map and reduce. 


\section{Conclusions}

In this paper, we have introduced a software restoration methodology for converting legacy-parallel applications into structured parallel code using parallel patterns. This ensures portability, maintainability and adaptivity of parallel code while maintaining, and sometimes even increasing, performance. We also presented transformations to eliminate ad-hoc pthread parallelism from legacy-parallel code, transformations that repair the code from bugs introduced by the elimination step, and, shape the code in order to patternise it. Furthermore, we evaluated out software restoration methodology on a number of realistic benchmarks and use-cases, demonstrating benefit in terms of gained performance, increased adaptivity, portability and maintainability. One of the limitations of the work is the fact that the transformations must be applied manually in their current form. It would be possible to take the transformations presented here and implement them into a semiautomatic refactoring tool, such as ParaFormance ${ }^{2}$, which is a semi-automatic tool for transforming sequential $\mathrm{C}$ and $\mathrm{C}++$ applications into parallel patterned versions. The refactorings presented in this paper would be implemented in terms of the pre-conditions and transformation rules of an Abstract Syntax Tree, both of which are described in this paper. Other similar refactorings to introduce patterns into $\mathrm{C}$ and $\mathrm{C}++$ applications have previously been described in $[7,8,9,27]$ and the restoration refactorings would take a similar direction. We will extend our methodology to take into account many other types of parallel patterns, including reduce, maps, stencil, etc. It's possible that we will see similarities and overlapping ideas in the restoration of different types of patterns. We will catalogue these commonalities in a future paper. We also intend on giving proofs of general soundness that our refactorings conform to their specification and do not change the program's functional behaviour. Proving soundness of refactorings is a complicated and challenging issue, but recent work in the use of Dependent Types [6] has allowed us to capture the soundness properties as part of the refactoring implementations. We envisage extending this to prove soundness of concurrency refactorings in a similar way. Lastly, we would like to explore optimisation of the restored application in other domains, such as energy optimisation. Once the code has been refactored into a structured, maintainable code base, it is possible to then apply different kinds of optimisations for energy, memory usage, etc. in a similar way to parallelisation.

Acknowledgements This work was generously supported by the EU Horizon 2020 project, TeamPlay (https://www.teamplay-h2020.eu), Grant Number 779882, and UK EPSRC Discovery, Grant Number EP/ P020631/1.

Open Access This article is licensed under a Creative Commons Attribution 4.0 International License, which permits use, sharing, adaptation, distribution and reproduction in any medium or format, as long as you give appropriate credit to the original author(s) and the source, provide a link to the Creative

\footnotetext{
$\overline{2 \text { http://www.paraformance.com/ }}$
} 
Commons licence, and indicate if changes were made. The images or other third party material in this article are included in the article's Creative Commons licence, unless indicated otherwise in a credit line to the material. If material is not included in the article's Creative Commons licence and your intended use is not permitted by statutory regulation or exceeds the permitted use, you will need to obtain permission directly from the copyright holder. To view a copy of this licence, visit http:// creativecommons.org/licenses/by/4.0/.

\section{References}

1. Aldinucci, M., Danelutto, M.: Stream Parallel Skeleton Optimization. In: PDCS, pp. 955-962 (1999)

2. Aldinucci, M., Danelutto, M., Kilpatrick, P., Torquati, M.: Fastflow: High-level and efficient streaming on multicore, chap. 13, pp. 261-280 (2017). 10.1002/9781119332015.ch13

3. Asanovic, K., Bodík, R., Demmel, J., Keaveny, T., Keutzer, K., Kubiatowicz, J., Morgan, N., Patterson, D.A., Sen, K., Wawrzynek, J., Wessel, D., Yelick, K.A.: A view of the parallel computing landscape. Commun. ACM 52(10), 56-67 (2009)

4. Axelsson, E., Claessen, K., Sheeran, M., Svenningsson, J., Engdal, D., Persson, A.: The design and implementation of feldspar-an embedded language for digital signal processing. In: The 22nd International Symposium on Implementation and Application of Functional Languages, IFL 2010, Lecture Notes in Computer Science, vol. 6647, pp. 121-136. Springer (2010)

5. Barwell, A.D., Brown, C., Hammond, K., Turek, W., Byrski, A.: Using program shaping and algorithmic skeletons to parallelise an evolutionary multi-agent system in Erlang. J. Comput. Inform. 35(4), 792-818 (2016)

6. Barwell, A.D., Brown, C., Sarkar, S.: Proof-carrying refactorings: sound refactoring for haskell via dependent types. In: International Conference on Functional Programming (ICFP), pp. 1-25. In Submission (2021)

7. Brown, C., Danelutto, M., Hammond, K., Kilpatrick, P., Elliott, A.: Cost-directed refactoring for parallel Erlang programs. Int. J. Parallel Programm. 42(4), 564-582 (2014)

8. Brown, C., Janjic, V., Barwell, A., García, J.D., MacKenzie, K.: Refactoring GrPPI: generic refactoring for generic parallelism in C++. Int. J. Parallel Programm. 48(4), 603-625 (2020). https://doi. org/10.1007/s10766-020-00667-X

9. Brown, C., Janjic, V., Hammond, K., Schöner, H., Idrees, K., Glass, C.W.: Agricultural reform: more efficient farming using advanced parallel refactoring tools. In: Euromicro International Conference on Parallel, Distributed and Network-based Processing, PDP, pp. 36-43. IEEE Computer Society (2014)

10. Brown, C., Janjic, V., Barwell, A, Thomson, J, Lozano, R. C., Cole, M, Franke, B, Garcia-Sanchez, J.D., Astorga, D. D. R., MacKenzie, K.: A hybrid approach to parallel pattern discovery in C++. In: 2020 28th Euromicro International Conference on Parallel, Distributed and Network-Based Processing (PDP), pp. 187-191 (2020)

11. Burstall, R.M., Darlington, J.: A transformation system for developing recursive programs. J. ACM 24(1), 44-67 (1977)

12. Butenhof, D.R.: Programming with POSIX Threads. Addison-Wesley Longman Publishing Co. Inc., USA (1997)

13. Campbell, C., Miller, A.: A parallel programming with microsoft visual $\mathrm{C}++$ : design patterns for decomposition and coordination on multicore architectures, 1st edn. Microsoft Press (2011)

14. Cook, J.E., Wolf, A.L.: Discovering models of software processes from event-based data. ACM Trans. Softw. Eng. Methodol. 7(3), 215-249 (1998)

15. Corbett, J.C., Dwyer, M.B., Hatcliff, J., Laubach, S., Pasareanu, C.S., Robby, Zheng, H.: Bandera: extracting finite-state models from java source code. In: Proceedings of the 22nd International Conference on Software Engineering ICSE, pp. 439-448. ACM (2000)

16. Dagum, L., Menon, R.: Openmp: an industry-standard API for shared-memory programming. IEEE J. Comput. Sci. Eng. 5(1), 46-55 (1998) 
17. De Sensi, D., De Matteis, T., Torquati, M., Mencagli, G., Danelutto, M.: Bringing parallel patterns out of the corner: the P3ARSEC benchmark suite. ACM Trans. Architect. Code Optim. (2017). https://doi.org/10.1145/3132710

18. del Rio Astorga, D., Dolz, M.F., Fernández, J., García, J.D.: A generic parallel pattern interface for stream and data processing. Concurr. Comput. Practice Exp. 29(24), e4175 (2017). https://doi.org/10. $1002 / \mathrm{cpe} .4175$

19. del Rio Astorga, D., Dolz, M.F., Sánchez, L.M., García, J.D., Danelutto, M., Torquati, M.: Finding parallel patterns through static analysis in $\mathrm{C}++$ applications. Int. J. High-Performance Comput. (IJHPCA) 32(6), 779-88 (2018)

20. Dig, D.: A refactoring approach to parallelism. IEEE Softw. 28(1), 17-22 (2011)

21. Fowler, M.: Refactoring-improving the design of existing code. Addison Wesley Object Technology Series. Addison-Wesley, Boston (1999)

22. González-Vélez, H., Leyton, M.: A survey of algorithmic skeleton frameworks: high-level structured parallel programming enablers. Softw. Pract. Exp. 40(12), 1135-1160 (2010)

23. Gorlatch, S.: Domain-specific optimizations of composed parallel components. In: Domain-specific program generation, Lecture Notes in Computer Science, vol. 3016, pp. 274-290. Springer (2003)

24. Gorlatch, S., Wedler, C., Lengauer, C.: Optimization rules for programming with collective operations. In: Proceedings of the 13th International Parallel Processing Symposium on Parallel and Distributed Computing IPPS/SPDP, pp. 492-499. IEEE Computer Society (1999)

25. Griebler, D., Danelutto, M., Torquati, M., Fernandes, L.G.: Spar: A DSL for high-level and productive stream parallelism. Parallel Processing Letters 27(1), 1740005:1-1740005:20 (2017). https:// doi.org/10.1142/S0129626417400059

26. Hagedorn, B., Stoltzfus, L., Steuwer, M., Gorlatch, S., Dubach, C.: High performance stencil code generation with lift. In: Proceedings of the International Symposium on Code Generation and Optimization, CGO, pp. 100-112. ACM (2018)

27. Janjic, V., Brown, C., Mackenzie, K., Hammond, K., Danelutto, M., Aldinucci, M., García, J.D.: RPL: A domain-specific language for designing and implementing parallel $\mathrm{C}++$ applications. In: Proceedings of the 24th International Conference on Parallel, Distributed and Network-based Processing, PDP, pp. 288-295. IEEE Computer Society (2016)

28. Kennedy, K.: A survey of data flow analysis techniques. In: Muchnick, S.S., Jones, N.D. (eds.) Program Flow Analysis, pp. 5-54. Prentice-Hall, Englewood Cliffs (1981)

29. Li, H., Thompson, S.J.: Safe concurrency introduction through slicing. In: Proceedings of the Workshop on Partial Evaluation and Program Manipulation, PEPM, pp. 103-113. ACM (2015)

30. Matsuzaki, K., Kakehi, K., Iwasaki, H., Hu, Z., Akashi, Y.: A fusion-embedded skeleton library. In: Euro-Par, Lecture Notes in Computer Science, vol. 3149, pp. 644-653. Springer (2004)

31. McCabe, T.J.: A complexity measure. IEEE Trans. Softw. Eng. 2(4), 308-320 (1976)

32. Mens, T., Tourwé, T.: A survey of software refactoring. IEEE Trans. Softw. Eng. 30(2), 126-139 (2004)

33. Microsoft: Visual Studio IDE: https: //visualstudio.microsoft.com/vs/. (2019)

34. Reyes, R., Lomüller, V.: SYCL: Single-source C++ accelerator programming. In: Proceedings of the International Conference on Parallel Computing (PARCO), Advances in Parallel Computing, vol. 27, pp. 673-682. IOS Press (2015)

35. TBB (Intel Threading Building Blocks). In: Encyclopedia of Parallel Computing, p. 2029. Springer (2011)

36. The Eclipse Foundation.: Eclipse-an open development platform (2009). http:// www.eclipse.org

37. Yu, Y., Wang, Y., Mylopoulos, J., Liaskos, S., Lapouchnian, A., do Prado Leite, J.C.S.: Reverse engineering goal models from legacy code. In: 13th IEEE International Conference on Requirements Engineering (RE 2005), 29 August-2 September 2005, Paris, France, pp. 363-372. IEEE Computer Society (2005)

Publisher's Note Springer Nature remains neutral with regard to jurisdictional claims in published maps and institutional affiliations. 